El movimiento estudiantil santafesino y sus estrategias de intervención colectiva en tiempos de lucha por la reforma universitaria y la creación de la Universidad Nacional del Litoral (1918 y 1919)

\title{
El movimiento estudiantil santafesino y sus estrategias de intervención colectiva en tiempos de lucha por la reforma universitaria y la creación de la Universidad Nacional del Litoral (1918 y 1919)
}

\author{
The Santa Fe student movement and its collective intervention \\ strategies in times of university reform, and the creation of the \\ Universidad Nacional del Litoral (1918 and 1919)
}

\author{
Eliana Bertero \\ Universidad Nacional del Litoral, \\ Programa Historia y Memoria \\ elibertero@gmail.com
}

\begin{abstract}
José Larker
Universidad Nacional del Litoral

Centro de Estudios Sociales Interdisciplinarios del Litoral (CESIL)

joselarker@yahoo.com.ar
\end{abstract}

\section{Resumen}

La Universidad Nacional del Litoral fue creada en 1919, fecha que coronó un proceso iniciado en 1912 con la movilización de los estudiantes universitarios santafesinos, en reclamo por una universidad nacional. Sus ideas y acciones lograron sumar a diversos actores políticos vinculados a un conjunto de asociaciones cívicas, culturales y políticas del arco liberal, como el Centro de Libre Pensamiento, la Sociedad Cosmopolita, la Logia Armonía y el Ateneo Popular, entre otros. Entre 1918 y 1919, los reclamos por la nacionalización se vieron impulsados por los acontecimientos que se sucedieron en Córdoba. En este contexto, el movimiento estudiantil santafesino definió estrategias específicas de intervención en la esfera pública, continuando con un ciclo de protestas que incluyó movilizaciones callejeras, conferencias en los locales de las federaciones obreras y bibliotecas populares, así como la huelga desplegada entre mayo y octubre de 1919.

Atendiendo a lo expresado, el trabajo tiene por objetivo reconstruir las formas de intervención pública de los estudiantes universitarios santafesinos durante la segunda década del siglo XX. Particularmente, la experiencia de la huelga estudiantil de 1919, explorando las demandas, los repertorios discursivos y de acción y la construcción de redes de solidaridad. 


\title{
Eliana Bertero y José Larker
}

\author{
Palabras clave \\ movimiento estudiantil; reformismo; demandas; repertorios de acción; Universidad \\ Nacional del Litoral
}

\begin{abstract}
Universidad Nacional Litoral was created in 1919, date that crowned a process initiated in 1912 with the mobilization of the Santafesinianuniversity students, who were reclaiming a national university. Their ideas and actions managed to add various political actors linked to a set of civic, cultural and political associations of the liberal arc, such as the Centro de Libre Pensamiento, the Sociedad Cosmopolita, the Logia Armonía, and the Ateneo Popular, among others. Between 1918 and 1919, the claims for nationalization were driven by the events that took place in Córdoba. In this context, the Santafesinian student movement defined specific intervention strategies in the public sphere, continuing with a cycle of protests that included street demonstrations, lectures in the premises of the workers' federations and popular libraries, as well as the strike deployed between May and October of 1919.

In response to the above, this work aims to reconstruct the forms of public intervention of Santafesinian university students during the second decade of the twentieth century. Particularly, the experience of the student strike of 1919, exploring the demands, the discursive and action repertoires and the construction of solidarity networks.
\end{abstract}

\section{Keywords}

students' movement; reformism; demands; action repertoires;Universidad Nacional del Litoral.

\section{La Universidad de Santa Fe y los comienzos de las demandas por la nacionalización}

Desde la creación de la Universidad de Santa Fe en 1890, la ciudad capital contaba con una casa de estudios superiores ${ }^{1}$. Por entonces, el Colegio de la Inmaculada Concepción era la única institución que expedía títulos de bachiller que habilitaban el ingreso a la misma, situación que se mantuvo hasta la creación del Colegio Nacional, en 1906. Dicha universidad constituía un emprendimiento de carácter provincial, vinculado a los grupos dirigentes e intelectuales que buscaban

\footnotetext{
1En 1890 se creó la universidad provincial, teniendo como antecedente a las facultades mayores anexas al Colegio de la Inmaculada Concepción, establecidas por ley provincial en 1868. Si bien desde 1871 se comenzó a hablar en la legislatura de una posible universidad, recién en 1889 se concretó un proyecto para la creación de la Universidad de Santa Fe. Desde ese momento, la nueva Casa de Altos Estudios dependería política y económicamente del Estado provincial y tendría por objeto formar abogados. Si bien la ley de creación contemplaba la conformación de tres facultades -Derecho y Ciencias Sociales, Físico Matemática y Teología-, solo funcionó la primera hasta 1911, año en que fue creada la Escuela de Farmacia y Obstetricia. Así, en los primeros veinte años, la Facultad de Derecho representó la única opción educativa de la universidad provincial.
} 


\section{El movimiento estudiantil santafesino y sus estrategias de intervención colectiva en tiempos de lucha por la reforma universitaria y la creación de la Universidad Nacional del Litoral (1918 y 1919)}

controlar el desarrollo de las instituciones culturales y asegurar el acceso de sus miembros a las profesiones liberales. ${ }^{2}$ La Universidad funcionó como una de las instituciones que permitió dotar de profesionales a un estado provincial en permanente crecimiento. La creación de nuevos organismos, el desarrollo de los negocios y el aumento poblacional así lo demandaban. Sin embargo, debido al monopolio ejercido por el Estado nacional para expedir títulos habilitantes para el ejercicio de la profesión, la Universidad de Santa Fe ofrecía un diploma que solo era aceptado en las provincias de Corrientes, Entre Ríos y la misma Santa Fe. Esta situación generó, desde sus inicios, la preocupación por resolver el problema de la validez nacional de los títulos. ${ }^{3}$

A comienzos de la década de 1910, a este problema, se sumaron fuertes críticas a la institución: a la orientación tradicional de la enseñanza, a los vínculos que mantenía con la iglesia católica y a la persistencia de un núcleo reducido en la dirección de la universidad.

En este contexto, las consignas de nacionalización se articularon con cuestionamientos que incluían desde las críticas al positivismo y la apelación a la laicización de la educación, hasta los debates sobre la cuestión regional. La incidencia de esos planteos al interior de la institución contribuyó a que se alinearan dos grupos en función de dos proyectos alternativos: uno que sólo demandaba la nacionalización de los títulos, mientras que el otro apelaba a la creación de una nueva universidad, entendida en clave reformista.

En torno al primero, concurrieron los sectores más tradicionales y conservadores de la universidad provincial, en su mayoría profesores, aunque contaba con el apoyo de estudiantes católicos de la facultad de derecho así como también del Colegio de la Inmaculada Concepción. El reclamo por la validez nacional de los títulos permitía resolver la situación de los abogados egresados de la universidad provincial en todo el ámbito nacional, sin modificar el modelo institucional. Como mencionamos anteriormente, la Universidad Provincial constituía un espacio privilegiado para la formación y reproducción de las elites locales, cuyos miembros habían consolidado su hegemonía en las cátedras y en el gobierno de la universidad. La posibilidad de ocupar estas posiciones jerárquicas obedecía a solidaridades legitimadas en lazos familiares, alianzas matrimoniales,

\footnotetext{
2 Osvaldo Graciano, Entre la torre de marfil y el compromiso político. Intelectuales de izquierda en la Argentina 1918-1955, Bernal, editorial UNQ, 2008.

${ }^{3}$ Desde diferentes ámbitos se llevaron a cabo gestiones para lograr la validez nacional de los títulos que expedía la Universidad de Santa Fe. En 1902, los diputados José Galeano y Nicasio Oroño presentaron el primer proyecto que proponía la nacionalización de los títulos pero no lograron tener éxito. En 1909, luego de varios años de gestión, el entonces Ministro de Justicia e Instrucción Pública de la Nación, firmó el Decreto del Poder Ejecutivo Nacional por el que se reconocían los títulos expedidos por la Universidad de Santa Fe. Véase José Larker, "El movimiento estudiantil universitario santafesino: acción y participación en el proceso de creación de la Universidad Nacional del Litoral", en Natalia Vega y Luciano Alonso (Compiladores), Lugares de lo colectivo en la historia local. Asociaciones, trabajadores y estudiantes de la zona santafesina. Santa Fe, María Muratore Ediciones, 2017.
} 


\section{Eliana Bertero y José Larker}

amistad, camaradería y fidelidades políticas, que trascendían el marco local y regional. Asimismo, la trama de relaciones en la que los actores participaban se veía consolidada a través de una sociabilidad específica que se construía en torno a aquellos valores ${ }^{4}$.

El segundo grupo, aglutinaba a estudiantes, profesores, políticos, muchos de ellos miembros activos del Centro de Libre Pensamiento, que reivindicaban los principios del reformismo liberal. Quienes se inscribían en esta corriente sostenían posiciones frecuentemente anticlericales. Sus posturas reformistas en lo social se basaron en el legalismo, o la convicción de que la política parlamentaria era el ámbito propicio para la búsqueda de soluciones al conflicto social y, por otro lado, en un cientificismo que acentuaría la importancia de las ciencias sociales como guía de la política estatal. ${ }^{5}$ En buena parte, el espíritu reformista se centró en la necesidad de transformar las instituciones y los hábitos políticos del país, sumidos en una crisis moral generalizada. Otros pusieron el acento en la necesidad de superar el "sórdido materialismo" y el "insaciable mercantilismo" imperante en la sociedad argentina. En definitiva, para estos liberales la reforma social y política era parte de un proceso

\footnotetext{
${ }^{4}$ En este sentido, la trayectoria de Zenón Martínez, por mencionar alguna, da cuenta del universo de prácticas y valores compartidos por la elite política local. Había nacido en Tulumba, provincia de Córdoba, el 24 de junio de 1857. Hijo de familias criollas, inicio sus estudios de derecho en las Facultades Mayores del Colegio Inmaculada y los completó en la Universidad de Córdoba, graduándose como Doctor en Jurisprudencia en 1882. Al cabo de tres años volvió a Santa Fe, abrió su estudio de abogado y poco después fue designado Juez de Menores y luego Juez del Crimen de Rosario. Un año más tarde se trasladó a la ciudad de Santa Fe para desempeñarse como Juez en lo civil y comercial y en 1887 pasó a ocupar una vocalía en el Superior tribunal de Justicia. No pasaría mucho tiempo para que fuera electo miembro de la convención constituyente que reformó la carta fundamental provincial en 1890. Concluida la reforma constitucional fue elegido Diputado y Senador provincial entre 1890 y 1893, pasando luego a ocupar la presidencia de la Corte Suprema de Justicia, función que desempeñaría desde 1894 hasta 1900. Pero Martínez no solo se dedicó a ocupar estrados en ámbitos tribunalicios o legislativos sino que desde el momento en que se creó la Universidad de Santa Fe en 1890, trabajó en ella desarrollando distintas funciones. Hasta 1911 estuvo a cargo de la cátedra de Derecho Romano. Además fue decano de la Facultad de Derecho y Ciencias Sociales y Rector de la universidad provincial en cuatro períodos: 1894/97, 1897/1900, 1900/03 y 1918/19. Martínez puede definirse como un miembro de la elite notabiliar santafesina. La Universidad de Santa Fe y la trayectoria de Zenón Martínez se aborda en un trabajo anterior. José Larker, "La Universidad de Santa Fe, la formación de abogados y sus concepciones acerca del Derecho". Ponencia presentada en las I Jornadas de Historia de la Universidad en la Argentina, Santa Fe, Universidad Nacional del Litoral, 2008. Sin lugar a dudas, la condición de abogado, juez, convencional constituyente, legislador, rector, decano y catedrático de la Universidad de la Universidad de Santa Fe, lo posicionaron como miembro de los grupos que controlaron el poder en la provincia entre las décadas finales del siglo XIX y las primeras del siguiente. Ocupó todas las posiciones jerárquicas institucionales y del ámbito político, lugares legitimados en lazos de solidaridad y en los principios y valores de la sociabilidad de la elite notabiliar local. Luk Boltanski, L'espacepositionnel. Multiplicité des positions institutionnelles et habitus de clase. En Revuefran ̧aise de sociologie, XIV, 1973. Traducción Nora Vega.

5Eduardo Zimmermann, "Los intelectuales y el reformismo liberal", en Desarrollo Económico, № 124, 1992. pp. 545-564. Para analizar la trama política santafesina, así como las ideas y preocupaciones de los grupos dirigentes del ámbito liberal, véase Bernardo Carrizo, "Temas y debates en tiempos de cambio. La legislatura de Santa Fe, 1912-1916". XII Jornadas Interescuelas/Departamentos de Historia. Facultad de Humanidades y Centro Regional Universitario Bariloche. Universidad Nacional del Comahue, San Carlos de Bariloche, 2009.
} 


\section{El movimiento estudiantil santafesino y sus estrategias de intervención colectiva en tiempos de lucha por la reforma universitaria y la creación de la Universidad Nacional del Litoral (1918 y 1919)}

de regeneración más amplio que se extendía desde la moral pública hasta los cambios institucionales.

Los liberales reformistas santafesinos, entre otras críticas, consideraban que las instituciones educativas locales existentes en los albores del XX constituían un anacronismo, fundamentalmente en un "pueblo que venía realizando los destinos prácticos de un cosmopolitismo de puertas abiertas". Estas instituciones representaban "un dique a la evolución rápida de la ciudad, así como a las costumbres e ideas modernas que, el progreso y la constante y abundante inmigración, traían". ${ }^{6}$ Desde esta perspectiva, la cuestión universitaria local se constituye en un espacio de disputas y conflictos, que excede a las demandas y reivindicaciones académicas e incluye la discusión sobre el modelo de sociedad. Estos grupos intervinieron decididamente en la esfera pública local a través de diversas acciones, entre las cuales la experiencia asociativa y los mítines se constituyeron en prácticas privilegiadas.

\section{El movimiento estudiantil universitario y las formas de acción colectiva en el proceso de creación de la UNL}

En 1912, la Federación de Estudiantes de Santa Fe -que agrupaba a estudiantes universitarios y secundarios y había sido creada a mediados de ese añoconvocó a un mitin bajo la consigna "pro nacionalización". Uno de los detonantes de esta movilización fue el hecho de que en ese mismo año la Corte Suprema de Justicia de la Nación desconociera el decreto del Poder Ejecutivo Nacional de 1909 que otorgaba validez nacional a los títulos expedidos por la universidad provincial. La organización del mitin y la invitación a los ciudadanos de Santa Fe y Paraná ocasionó una gran repercusión pública medida en la participación de amplios sectores, entre los que se encontraban el entonces gobernador Manuel Menchaca, además de profesores, directores, corresponsales de diarios, varias asociaciones, vecinos y delegaciones de Esperanza, Coronda, Corrientes y Paraná.

Estas instituciones se encontraban en la base de un movimiento liberal que intervino en instancias vinculadas a las luchas por la laicización de la educación, como la creación del Colegio Nacional y la Escuela Normal. Si estos proyectos, por

\footnotetext{
${ }^{6}$ Es Manuel Menchaca, gobernador radical electo en 1912 y miembro activo de las asociaciones liberales, quien en una mirada retrospectiva, evoca el ambiente cultural de la época: "La formación cultural, en Santa Fe, al iniciarse el siglo [XX], estaba dirigida, en todas sus manifestaciones por el Colegio de la Inmaculada Concepción, establecimiento educacional que venía, desde tiempo atrás, desarrollando una acción encomiable, dentro de sus principios, que no eran los adecuados ni diversos que imponía el momento ... El instituto seleccionaba su alumnado entre los jóvenes de familia de tradición o extranjeros adinerados, tanto de Santa Fe, como de Entre Ríos y Córdoba, con la visión de hacer ex - alumnos influyentes en los destinos de la provincia y del país." Manuel Menchaca, "La Universidad Nacional del Litoral. Antecedentes de su creación", en Revista Universidad, N 48 , Santa Fe, UNL, 1961, pp.145-146. En un tono similar Raúl Villarroel señalaba: una "fuerza inercial” provenía del poder reaccionario y conservador, es decir, la tendencia religioso-clerical estrechamente vinculada a los jesuitas. Raúl Villarroel "Santa Fe", RACP, T. VII, septiembre 1913.
} 


\section{Eliana Bertero y José Larker}

un lado, aglutinaron a los hombres de ideas liberales, por el otro, congregaron a una oposición que, como ya mencionamos, se identificada con los sectores más conservadores de la sociedad santafesina. En tales circunstancias, la cuestión universitaria se superpuso a una disputa ideológica y cultural, cuando en 1912 el diputado Alcides Greca presentó un proyecto de educación primaria laica, instalando el debate educativo en la legislatura provincial. En esa disputa, los estudiantes santafesinos, tramaron vínculos con profesores y políticos liberales, en su mayoría del partido radical, comenzando a reunirse en comités y centros culturales e intelectuales desde donde delinearon los principios, valores y reglas que inspiraron el proyecto de modernización social y cultural para la región.

La apelación a la creación de una nueva casa de estudios superiores se articulaba con un conjunto de reivindicaciones vinculadas a las ideas reformistas. Portador de esas ideas, Alejandro Grüning Rosas se constituyó en el principal interlocutor de los estudiantes, iniciando una fuerte campaña pro Universidad del Litoral que logró amplios consensos en el ámbito local y regional. Estas adhesiones respondían en parte a los vínculos que muchos estudiantes tenían con las organizaciones liberales de Santa Fe, Paraná e incluso Rosario. Un ejemplo de ello es el propio Grüning Rosas, joven rosarino que en la década 1910 se constituyó en una figura relevante del movimiento estudiantil santafesino, teniendo proyección nacional como dirigente de la Federación Universitaria Argentina. En 1915 se desempeñaría como secretario de redacción de La Palabra, periódico ligado al partido radical, y en 1917 asumiría como director. Además fue miembro del Centro de Libre Pensamiento de Santa Fe y de la Sociedad Cosmopolita. ${ }^{7}$ Sus discursos expresaban principios y valores construidos en esos espacios de sociabilidad y producto de los vínculos discipulares que mantenía con librepensadores como Raúl Villarroel, Luis Bonaparte y con reconocidos dirigentes radicales como Manuel Menchaca y Alcides Greca. Grüning Rosas, en el mitin de 1912 expresaba:

La nacionalización traerá la reforma de lo que sea menester reformar, para que nuestra Universidad, hasta hoy mirada, con razón o sin ella, en Buenos Aires y otras partes, como un anacronismo inútil y estéril, pueda imponerse ante la consideración nacional, colocarse a la altura de la universidades modernas, ponerse más en contacto con los elementos vivos de la provincia y del país y dar en suma todos los beneficios intelectuales que es dable esperar de un instituto de estudios superiores que debe ser guión y nunca rémora del progreso de la patria. ${ }^{8}$

De esa manera, el discurso enunciaba los fundamentos de un movimiento reformista que logró establecer acuerdos sobre los contenidos del proyecto involucrando a los estudiantes y "las fuerzas vitales, todos los elementos populares

\footnotetext{
${ }^{7}$ Museo Histórico UNL. Militantes de la universidad reformista... hacedores del cambio. Santa Fe, Universidad Nacional del Litoral, 2008.

${ }^{8}$ Diario La Nación, 9 de setiembre de 1912, cit. en Alejandro Grüning Rosas, Creación de la Universidad Nacional del Litoral. Crónica retrospectiva. Santa Fe, Imprenta de la Universidad. 1940, p. 30.
} 


\title{
El movimiento estudiantil santafesino y sus estrategias de intervención colectiva en tiempos de lucha por la reforma universitaria y la creación de la Universidad Nacional del Litoral (1918 y 1919)
}

y progresistas de Santa Fe". ${ }^{9}$ Sus principales tópicos referían a un modelo de universidad de carácter regional que, en consonancia con las necesidades propias de la modernización social, rompiese con la universidad tradicional. Entre otros puntos, el programa de la juventud universitaria, planteaba la necesidad de conformar una federación de Estudiantes que trascendiera el ámbito local y construyera lazos de solidaridad a nivel nacional. Asimismo, se apelaba a la conformación de un frente unido de estudiantes y trabajadores:

\begin{abstract}
Esta Federación Estudiantil, formada por la juventud estudiosa que ha de ser mañana directora lógica de los destinos colectivos, es hoy local, mañana será provincial y más tarde nacional, pues entendemos que una de las más altas misiones actuales es tender, por todos los medios lícitos posibles, a borrar antagonismos extemporáneos de regionalismos o de clases, a formar la unidad nacional, en que debe figurar por igual el obrero que es brazo, sin el cual la idea es estéril, y estudioso, que es la fuerza, sin la cual el brazo se quebranta. ${ }^{10}$
\end{abstract}

Planteado en estos términos, el proyecto de los estudiantes santafesinos abrió un espacio de pujas y conflictos que desbordó el ámbito universitario irrumpiendo en la esfera pública. La conformación de comités y la organización de mítines se constituyeron en formas de intervención privilegiada del movimiento estudiantil santafesino. Desde allí demandaron y presionaron a las autoridades con el fin de producir resultados políticos. Particularmente, en el conflicto entablado alrededor de la creación de la nueva universidad se cruzaban diferentes discursos que iban desde el espiritualismo arielista ${ }^{11}$-en oposición a lo que se definía como la modernización materialista- hasta el anticlericalismo, pasando por una consideración de los problemas académicos específicos. Asimismo, la discusión abierta por las demandas de nacionalización alentó los cuestionamientos a la orientación profesionalista, como a la democratización del modelo tradicional conservador.

Los años 1914 y 1915 se constituyeron en momentos clave para el proyecto de universidad nacional, pues se crearon los Centros de Estudiantes de Derecho y de Farmacia y Obstetricia, en sustitución de la Unión Universitaria que los nucleaba hasta ese momento. En 1915, al interior de estas organizaciones, se impusieron los grupos reformistas, fortalecidos con el aporte de las primeras promociones de los colegios secundarios laicos, recientemente creados en la ciudad de Santa Fe, como

\footnotetext{
9 Ídem, p. 29

10 Ídem, p. 30

11 Sobre la recepción y circulación del juvenilismo condensado por Rodó en el Ariel, véase Natalia Bustelo, La reforma universitaria desde sus grupos y revistas: Una reconstrucción de los proyectos y las disputas del movimiento estudiantil porteño de las primeras décadas del siglo XX (1914-1928). Tesis de posgrado. Universidad Nacional de La Plata. Facultad de Humanidades y Ciencias de la Educación. En Memoria Académica. 2015. Disponible en:
} http://www.memoria.fahce.unlp.edu.ar/tesis/te.1307/te.1307.pdf 


\section{Eliana Bertero y José Larker}

el Colegio Nacional. Estos centros de estudiantes propusieron la creación de facultades en las provincias de Santa Fe y Entre Ríos.

Con el objetivo de impulsar un proyecto de carácter regional, se pusieron en contacto con estudiantes de Rosario y Paraná, estimulando la creación de Federaciones estudiantiles en ambas ciudades. En setiembre de 1915, se realizó un congreso de estudiantes en la Biblioteca Popular de Paraná, con la asistencia de delegados de dicha ciudad, de Santa Fe, Buenos, Rosario, San Nicolás, Salta, Tucumán y Catamarca. Bajo el lema "patria, ciencia, trabajo y libertad", el congreso declaró que la creación de una universidad nacional era una necesidad impostergable. Los gobiernos de Santa Fe y Entre Ríos apoyaron la creación de la nueva universidad y el periódico El Diario de Paraná difundía las ideas del movimiento estudiantil. El proyecto sostenía la necesidad de una universidad con institutos en Santa Fe, Paraná y Rosario, con sede central en la primera. Establecía también que la orientación de la nueva institución debía seguir los lineamientos de las universidades modernas como las de La Plata y Tucumán en Argentina, Alemania, Estados Unidos y Gran Bretaña. Los estudiantes destacaban, además, "la conveniencia de integrar la nueva universidad litoralense con las bibliotecas Cosmopolita de Santa Fe, Argentina de Rosario y Popular de Paraná, como centros indicados para la extensión universitaria".12

La extensión de redes y construcción de solidaridades entre los estudiantes de diferentes ciudades de la región siguió siendo motivo de trabajo por parte de los jóvenes universitarios santafesinos. Respecto de ello, los contactos y reuniones entre las diferentes federaciones estudiantiles no dejaron de realizarse. El diario $L a$ Capital de Rosario, en su edición del día 28 de agosto de 1915, informaba sobre las actividades de los estudiantes rosarinos: "Ayer se reunió en sesión extraordinaria la comisión directiva de esta entidad con el objeto de tratar el interesante tópico de la creación de una universidad en el litoral. Asistieron a esta sesión los señores Domingo Sabaté y Emilio R. Tasada, presidente y secretario respectivamente del Centro de Estudiantes de Derecho de la ciudad de Santa Fe."13

En 1916 se formaron asociaciones para promover la creación de la nueva casa de estudios, instalando el proyecto en el Congreso Nacional. Es el caso del Comité Pro Universidad del Litoral creado en la Sociedad Cosmopolita ${ }^{14}$ e integrado, entre otros, por Alejandro Grüning Rosas, Raúl Villarroel, Luis Bonaparte, Manuel Menchaca, Enrique Mosca, Luis Reggiardo y Cristóbal Roca. Para actuar en Buenos Aires se fundó el Centro de Residentes Santafesinos, del que formaron parte Rodolfo Rivarola, Joaquín V. González, Tomás Cullen y Leopoldo Melo. Paralelamente, la

12Ídem, p.35.

${ }^{13}$ Alejandro Grüning Rosas. Creación de la Universidad Nacional del Litoral (Crónica Retrospectiva). Apéndice documental complementario, Buenos Aires, 1940, p. 39

${ }^{14}$ Cabe destacar que la Sociedad Cosmopolita de Socorros Mutuos fue fundada en 1883. Esta asociación de filiación liberal, tomó partido en la modernización de la ciudad constituyéndose en el púlpito desde donde un cúmulo de prácticas e ideas liberales adquirieron estado público. 


\section{El movimiento estudiantil santafesino y sus estrategias de intervención colectiva en tiempos de lucha por la reforma universitaria y la creación de la Universidad Nacional del Litoral (1918 y 1919)}

Federación Universitaria difundía sus ideas y acciones a través de la prensa local, como el diario Santa Fe. ${ }^{15}$

Quienes conformaban el comité Pro Universidad -estudiantes, profesores y dirigentes políticos- tenían una presencia estable en el entramado de instituciones y espacios de sociabilidad que intervenían públicamente de los debates y conflictos en torno a la cuestión de la universidad. ${ }^{16}$ Para completar, en parte la imagen de este entramado de relaciones que confluyeron en aquellos comités, resulta importante mencionar cómo figuras políticas relevantes ligadas, fundamentalmente al radicalismo, y reconocidos librepensadores -como el entonces gobernador Manuel Menchaca, Alcides Greca, Luis Bonaparte, Armando Antille y Enrique Mosca, entre otros- articularon acciones con los estudiantes universitarios.

Asimismo, la intervención de figuras que provenían también del ámbito académico, como Rodolfo Rivarola, mostraron los vínculos que los estudiantes universitarios santafesinos establecieron con algunos intelectuales y políticos de convicciones liberales reformistas. En octubre de 1914, invitado por el Centro de Estudiantes de Derecho, Rodolfo Rivarola ofrece una conferencia en Santa Fe, sobre Sáenz Peña y su obra democrática. En esa ocasión la Federación de Estudiantes le confía la redacción del primer proyecto de universidad de tipo regional, al que el propio Rivarola propone la denominación del Litoral. Este vínculo se sostiene, entre otros cosas, a través de una nutrida correspondencia que circula entre Rivarola y los estudiantes santafesinos. Las notas enviadas por Grüning Rosas, en calidad de presidente de la Federación Universitaria de Santa Fe, mostraban los lazos de solidaridad entre un reconocido intelectual y los estudiantes universitarios. En instancias de la visita de Rivarola a la ciudad de Santa Fe, los estudiantes decían: "Santa Fe necesita de esa clase de hombres; hacen falta sus visitas frecuentes, sus esfuerzos, ya que ellos redundarán, indiscutiblemente, en el adelanto general. Son sembradores; hay que cultivar; hay que educar, que modelar cerebros juveniles, haciendo obra para el mañana..."17 A mediados de 1915 la Federación estudiantil envía una nota a Rivarola advirtiendo las resistencias que encontraba el proyecto reformista al interior de la universidad provincial. 18 "El viaje del señor rector a ésa

\footnotetext{
${ }^{15}$ Entre julio y setiembre de 1919, la Federación Universitaria utilizó un órgano de difusión propio como La Gaceta Universitaria de Santa Fe.

${ }^{16}$ En la reunión en la que quedó constituido el comité, bajo la presidencia provisoria de Menchaca, se indicaba la conveniencia de que se invitara ampliamente, "sin ninguna distinción de colores partidistas o de otra especie, a la reunión que con carácter definitiva tendría lugar el viernes próximo a la 8.30, en el mismo local". Acta $N^{\circ} 1$ Comité Pro-Universidad Nacional del Litoral, Santa Fe, 26 de junio de 1916. Museo Histórico UNL "Marta Samatán".

${ }^{17}$ Alejandro Grüning Rosas. Creación de la Universidad Nacional del Litoral...Op. Cit., p.37

${ }^{18}$ Las actas del consejo superior dejan traslucir las posiciones conservadoras de las autoridades universitarias. En 1916, el consejero por Farmacia y Obstetricia Cristóbal Roca (perteneciente a los sectores reformistas) exige que el Consejo Superior defina su posición frente a los dos proyectos que pretendían dar solución al problema de la universidad. En esta instancia se formularon de la siguiente manera: gestionar la nacionalización de la universidad o propiciar la creación de la Universidad Nacional del Litoral. Este cuerpo se inclina por la primera, generando duras críticas en los estudiantes
} 


\section{Eliana Bertero y José Larker}

(Buenos Aires), creo que ha sido para trabajar por la validez nacional de los títulos de la Universidad Provincial de Santa Fe y no por la UNL. La juventud debe tener una aspiración y sostenerla y realizarla (...) Ella confía en la justicia y en la sinceridad y patriotismo de sus verdaderos maestros, que son los que señalan rumbos a las colectividades y engrandecen los pueblos".19

Por su parte, la prensa local y revistas prestigiosas del ámbito nacional, asumieron un rol protagónico como tribuna de los estudiantes reformistas. En este sentido, la Revista Argentina de Ciencias Políticas reflejaba a través de su corresponsal santafesino, Raúl Villarroel ${ }^{20}$, las disputas del contexto local:

...aquí las ideas nuevas hallan resistencia organizada y sistemática, que viene a mezclarse con las cuestiones políticas, económicas y demás. La tendencia tradicionalista, digámoslo así, cuenta aún con el núcleo "católico y dogmático", unido por lazos de religión, de parentesco, de intereses creados (...) la tendencia liberal y democrática militante, cuenta en cambio (...) con un núcleo organizado de intelectuales, pocos en número y de escaso valor monetario, pero activos, populares, estudiosos (...) organizados en el Centro de Libre Pensamiento, pronuncian discursos, organizan mítines, peticionan la sanción de leyes reformistas, protestan contra todo abuso autoritario y dogmático. ${ }^{21}$

Las cartas, telegramas y encuentros con reconocidos dirigentes políticos del radicalismo e intelectuales del arco liberal, evidenciaban también una novedosa estrategia de intervención de los universitarios en la esfera pública, principalmente la negociación para el logro de los consensos parlamentarios en favor del proyecto reformista. ${ }^{22}$ En 1917, las distintas organizaciones que se habían constituido para lograr la creación de la Universidad Nacional del Litoral, continúan con las negociaciones. Las reuniones y las comunicaciones entre el Comité Pro Universidad del litoral, el Centro de Residentes Santafesinos en Buenos Aires, así como de diversas instituciones santafesinas y rosarinas con los legisladores fueron frecuentes. El diputado Jorge R. Rodríguez presentó un Proyecto de ley para la Universidad de Santa Fe que contó con el apoyo del centro de estudiantes de la Escuela Industrial de Rosario, de vecinos de Santa Fe y Paraná y del Colegio de

universitarios como un acto para sostener el criterio conservador de la casa de estudios existentes. Santa Fe, Santa Fe, 29/07/1916, p. 2.

19 Nota de la Federación Estudiantil, 02/07/1915. En Alejandro Grüning Rosas. Creación de la Universidad Nacional del Litoral... Op. Cit. pp. 45 y 46

${ }^{20}$ Raúl Villarroel no era un simple cronista. Activo librepensador, miembro de la logia Armonía y del Comité Pro Universidad, se constituyó en vocero privilegiado de una fecunda lucha periodística. Sus columnas ponían de manifiesto una larga confrontación en la cual, librepensadores y masones embestían contra la iglesia católica haciéndola responsable, en este caso, de frenar la modernización y democratización de las instituciones.

${ }^{21}$ Raúl Villarroel, RACP, Tomo X, Buenos Aires, mayo de 1915, pp. 296-297.

${ }^{22} \mathrm{La}$ trayectoria del proyecto de creación de la UNL en el debate parlamentario, en el que se expresaron los diferentes posicionamientos políticos y regionales, puede verse en Eliana Bertero, "Entre la tradición y el reformismo. La cuestión universitaria santafesina en el debate público (19121919)". Ponencia presentada en el III Congreso Regional de Historia e Historiografía, Santa Fe, 2009. 


\section{El movimiento estudiantil santafesino y sus estrategias de intervención colectiva en tiempos de lucha por la reforma universitaria y la creación de la Universidad Nacional del Litoral (1918 y 1919)}

Abogados y Círculo Médico de Rosario. Sin embargo no se lograron avances significativos, quedando el proyecto sin la aprobación de la comisión. Al año siguiente, el conflicto abierto entre el estudiantado cordobés y las autoridades de la Universidad de aquella provincia, planteaba un nuevo contexto para el proyecto santafesino.

\section{El movimiento estudiantil santafesino, la reforma universitaria y la huelga de 1919}

Entre 1918 y 1919, las demandas por la creación de la nueva universidad y las acciones emprendidas por los estudiantes se profundizaron. Por entonces, el Centro de Estudiantes de Derecho se nutría de nuevos dirigentes estudiantiles, como Pablo Vrillaud, Mariano Tissembaum y Horacio Varela, entre otros. Estos estudiantes reunían ciertos rasgos singulares de los universitarios reformistas santafesinos. De modo casi excluyente, eran hijos de inmigrantes provenientes de los sectores medios, habían cursado sus estudios en el Colegio Nacional de Santa Fe, donde se conocieron y obtuvieron el título de bachiller en 1917 e ingresaron a la Universidad provincial en 1918. Tempranamente participaron de diversas asociaciones de la cultura y la política local como el Centro de Libre Pensamiento y el Ateneo Popular de Santa Fe, a través de las cuales se impulsó la creación de instituciones educativas laicas. Allí establecieron vínculos con profesores, periodistas y políticos que apoyaron las demandas de la juventud universitaria.

Sumado a las demandas locales, el conflicto de la universidad de Córdoba ${ }^{23}$ ocupó la esfera pública santafesina y tuvo eco en la prensa local y en la de circulación nacional, donde estudiantes, profesores y "hombres de la cultura" se pronunciaron ante el conflicto. Los círculos y centros de estudiantes católicos, organizados en una federación ${ }^{24}$, expresaron su adhesión pública a la elección de Antonio Nores como rector de la Universidad de Córdoba. Mientras, los grupos reformistas, no sin resistencias, intentaban reformar las estructuras de la institución universitaria local.

En el Primer Congreso de Estudiantes Universitarios realizado en Córdoba en 1918, el delegado por Santa Fe Ángel Caballero Martín ${ }^{25}$ introdujo el proyecto de

\footnotetext{
${ }^{23}$ Sobre el movimiento reformista de 1918 en Córdoba y el rol de los estudiantes, referimos al trabajo de Pablo Buchbinder ¿Revolución en los claustros?: la reforma universitaria de 1918, Buenos Aires, Sudamericana, 2008.

${ }^{24}$ En el mes de julio de 1918 se realiza un Congreso de los Centros de Estudiantes Católicos del país, con el objetivo de organizar una federación. El delegado estudiantil por Santa Fe, Manuel del Sastre es elegido presidente de dicha federación, que sanciona un proyecto de declaración por el cual se establece que la misión fundamental de los centros católicos es la formación integral de sus asociados. Santa Fe, Santa Fe, 15/07/1918, p.2.

${ }^{25}$ Además de Caballero Martín, la representación santafesina estaba compuesta por los estudiantes: Alejandro Gruning Rosas, Pablo Vrillaud, Horacio J. Varela, Mario R. Tissembaun, Juan A. Sanz, Octavio Martínez, Humberto Gambino y Raúl Zaballa.
} 


\section{Eliana Bertero y José Larker}

creación de la Universidad Nacional. Los estudiantes dieron apoyo a "la brega por la Universidad Nacional del Litoral, en la que aspiraban a aplicar la reforma anhelada." 26 Ello quedó reflejado en la resolución sancionada por el congreso el 24 de julio, en el que se recomendaba al Congreso Nacional "la pronta sanción del proyecto creando la Universidad Nacional del Litoral."27

Por su parte, las acciones emprendidas por los reformistas motivaron el apoyo de la prensa local, reivindicando, a través de sus columnas, la participación del movimiento estudiantil santafesino en la lucha por la reforma de la universidad. Al respecto, resulta ilustrativo un editorial en el que el diario Santa Fe reconstruye ese proceso iniciado en 1912:

Desde 1912, la juventud universitaria acompañada por los hombres de pensamiento (que lo son sin necesidad de ocupar cátedra universitaria), ha venido luchando por la reforma. Continuó impertérrita, a pesar de la guerra que contra ella abrieran las autoridades universitarias que a falta de sinceridad solo han empleado la astucia y recursos de mala ley para defender lo indefendible, para mantener únicamente posiciones presupuestarias acumuladas por parientes y socios, en tal forma, que los términos oligarquía y nepotismo no resultan mal utilizados, ni tampoco el de corda. Esas autoridades se opusieron a la reforma y la creación de la universidad nueva...28.

A comienzos de 1919, el proyecto de creación de la universidad adquiere nuevo impulso en el Congreso Nacional. En un contexto de creciente conflictividad, en el que los estudiantes santafesinos radicalizaron sus acciones, reclamando además de la nacionalización, la reforma de los estatutos. En 1914, la Universidad de Santa Fe había reformado los estatutos, estableciendo la elección del rector por una Asamblea Universitaria compuesta por académicos titulares, representantes de las facultades de Ciencias Jurídicas y Sociales y de Farmacia y Obstetricia. Sin embargo, en esta instancia, el movimiento estudiantil exige la adopción de los estatutos reformistas de la Universidad de Buenos Aires. Fundamentalmente, buscaban suprimir el carácter vitalicio de los académicos y reconocer el derecho a tener representación en los consejos.

En abril de 1919, la Federación de Estudiantes Universitarios declaró la necesidad de una modificación substancial del gobierno de la universidad en su "composición y orientaciones"29.En este clima, el Consejo Superior adoptó los estatutos reformistas de la UBA. Con ello, las autoridades de la universidad decían proponerse encuadrar "el régimen de su gobierno dentro de las bases que han de

\footnotetext{
${ }^{26}$ José Carmelo Busaniche, Universidad Nacional del Litoral, Antecedentes y Creación, texto mecanografiado y sin fecha, p. s/n.

${ }^{27}$ Gabriel Del Mazo, La Reforma Universitaria, documentos relativos al movimiento estudiantil en las universidades de Córdoba y Buenos Aires, Federación universitaria de Buenos Aires, Buenos Aires, 1927, Tomo llI, p. 196.

${ }^{28}$ Santa Fe, Santa Fe, 09/09/1918, p. 2.

${ }^{29}$ Santa Fe, Santa Fe, 7/04/1919, p. 2.
} 


\section{El movimiento estudiantil santafesino y sus estrategias de intervención colectiva en tiempos de lucha por la reforma universitaria y la creación de la Universidad Nacional del Litoral (1918 y 1919)}

regirla cuando se forme con sus elementos la esperada universidad nacional"30. Sin embargo el artículo 5o establecía que la reorganización quedaba en manos del entonces rector, Zenón Martínez y este hecho agravó el conflicto.

El 8 mayo de 1919, en la asamblea reunida en el local de la Sociedad Cosmopolita, la Federación Universitaria de Santa Fe, exigió la renuncia del rector y de todo el profesorado y solicitó al Poder Ejecutivo Provincial el nombramiento de un representante que tuviera a su cargo la reorganización. ${ }^{31}$

Ante la negativa de las autoridades a renunciar, los estudiantes convocaron a una huelga que se prolongó hasta octubre, al tiempo que desplegaron otras acciones en defensa del proyecto reformista: conferencias, mítines y gestiones ante los poderes Ejecutivo y Legislativo Nacional.

El discurso del dirigente estudiantil Pablo Vrillaud ${ }^{32}$, expresaba los términos de las demandas:

Pedimos estatutos dignos y profesorado capaz. Vivimos días bellos de acción y pensamiento. Reivindicamos con este gesto un concepto que se nos discutía. Ante la pasividad de academias desprestigiadas, resoluciones enérgicas adquieren fuerza simbólica. Es la muerte de un régimen (...) como en Córdoba hay defensores de lo anacrónico: minoría familiar, guardianes de sus papás catedráticos; esto, en vez de desalentarnos nos conforta. ${ }^{33}$

Este discurso y los hechos que se sucedieron durante estos días, pusieron de manifiesto las tensiones y disputas al interior del movimiento estudiantil. Mientras la Federación Universitaria realizaba la asamblea en la Sociedad Cosmopolita, más de cincuenta alumnos de la Facultad de Derecho y Escuela de Notariado y Procuración se reunieron en la casa de estudios y constituyeron un centro llamado Unión Universitaria, cuyo presidente y secretario eran los estudiantes César Gauchat y Juan L. Rueda. Este grupo manifestó públicamente su apoyo a las autoridades como legítimas reorganizadoras de la universidad y recomendaron la asistencia a clases. Al respecto el diario Santa Fe, refiriéndose al momento de iniciarse la asamblea convocada por la Federación expresaba: "se temía que la asistencia fuera insuficiente porque el grupo que podríamos llamar conservador, secundando al grupo netamente católico, estaba en los claustros dispuestos a concurrir a clases." 34

\footnotetext{
30 Ángel Caballero Martín, La Universidad de Santa Fe, Imprenta de la Universidad, Santa Fe, 1931, p. 107.

${ }^{31}$ Santa Fe, Santa Fe, 9/05/1919, p. 2.

${ }^{32}$ En 1918 fue enviado como delegado al Congreso Nacional de estudiantes de Córdoba y elegido presidente de la Federación Universitaria de Santa Fe.

33 Alejandro Grüning Rosas, Creación de la Universidad Nacional del Litoral. Apéndice documental complementario... Op. Cit. p. 62.

${ }^{34}$ Santa Fe, Santa Fe, 9/05/1919, p.2.
} 


\section{Eliana Bertero y José Larker}

Al día siguiente de la asamblea realizada en la Sociedad Cosmopolita la Federación emitió un comunicado en el que manifestaba mantener las decisiones tomadas y gestionar ante las autoridades provinciales la intervención de la universidad. Sumado a ello, según se registra en el diario de campaña de los estudiantes de Santa Fe y en el diario Santa Fe, "la Federación universitaria expulsa de su seno a los estudiantes que constituyen la <Unión Universitaria>, ya que las resoluciones adoptadas por esta agrupación reflejan que marcha a impulso de indicaciones de las autoridades..." 35 .A través del mismo comunicado, se planteaba la necesidad de generar lazos de solidaridad y/o adhesiones con otras federaciones, con sectores liberales reformistas del arco político y de la sociedad civil y con las organizaciones obreras de la ciudad.

El conflicto se fue profundizando, el 11 de mayo, en solidaridad con los estudiantes universitarios, los alumnos del Colegio Nacional no entraron a clases y se hizo presente la policía y el escuadrón de seguridad para evitar posibles incidentes. Con todo, se produjeron "algunas escaramuzas a manos limpias y hasta la exhibición de un Smith Wesson [...]. En el resto del día los grupos de ambos centros acantonados a pocos metros de distancia continuaron el fogoneo de gritos e insultos más o menos eufóricos." ${ }^{36} \mathrm{Al}$ medio día la Federación Universitaria festejó y la ciudad se enteró de ello a través de los estruendos producidos por el estallido de bombas. El alboroto se debía a un "supuesto decreto ministerial." En este contexto, el Rector Zenón Martínez presentó su renuncia y tras él, también lo hicieron la mayoría de los docentes, quedando solo algunos que al momento de presentarse la intervención debieron efectivizarla. El Decreto de intervención publicado por el diario Santa Fe planteaba que era necesario reorganizar las autoridades de la Universidad de Santa Fe, de acuerdo con los estatutos que se ponían en vigencia a partir de ese día, que las medidas que se tomaban obedecían a las solicitudes de los alumnos y los catedráticos y que las mismas estarían bajo la conducción del Ministro de Instrucción Pública y agricultura. En función de esas consideraciones, se declaró intervenida la casa de altos estudios. ${ }^{37}$

El mismo día entraron en funciones los nuevos delegados del Centro de Estudiantes de Derecho y se renovaron las autoridades de la Federación Universitaria. La nueva comisión quedó constituida por Pablo Vrillaud y J. A. Sanz como presidentes, Augusto Morisot y J. Rubio como secretarios, E. Dottoni asumió el cargo de tesorero y los vocales fueron: Horacio Gambino, Alejandro Gruning Rosas, Ignacio González, J. Pietranera, P. Candioti, A. Rotman y T. Ramella. En el acto la Federación emitió una declaración realizando una evaluación de las acciones llevadas a cabo por la Unión Universitaria. Entendían que ese grupo expresaba "contradicciones manifiestas entre lo que realizan y lo que publican", por lo que

\footnotetext{
${ }^{35}$ Diario de Campaña de los estudiantes de Santa Fe. En Alejandro Grüning Rosas, Op. Cit. p. 62.

${ }^{36}$ Santa Fe, Santa Fe, 14/05/1919, p. 2. Cuando se refiere a "un Smith Wesson" está haciendo alusión a una pistola de origen norteamericano.

${ }^{37}$ Ibídem.
} 


\section{El movimiento estudiantil santafesino y sus estrategias de intervención colectiva en tiempos de lucha por la reforma universitaria y la creación de la Universidad Nacional del Litoral (1918 y 1919)}

consideraban necesario dar a conocer los motivos del conflicto. ${ }^{38}$ Con ese objetivo, durante el conflictivo mes de mayo la Federación Universitaria realizó una serie de conferencias en los locales de la federación obrera, en el centro de empleados de comercio y en la Federación Ferroviaria. ${ }^{39}$

Al día siguiente tuvo lugar el acto de toma de posesión de las autoridades interventoras. La prensa local recreaba el clima de los acontecimientos: "la federación universitaria y la Unión Universitaria se congregan en sus locales frente a la facultad", de donde se disparaban bombas de estruendo, acompañadas de aplausos y consignas a favor y en contra del rector Zenón Martínez. Se destaca también que "al local de la Federación concurren los alumnos del colegio nacional que han hecho causa común con los universitarios en huelga."40

En el salón de actos de la universidad Zenón Martínez, acompañado de un grupo de profesores y amigos personales esperó la llegada del Interventor. Según informa el Santa Fe "La sala de actos estaba repleta de concurrencia selecta, entre la que figuraban no pocos profesores de la misma que no participaban del círculo del rector. La Federación Universitaria y la Unión Universitaria estaban representadas por delegaciones numerosas." ${ }^{41} \mathrm{Al}$ momento de los discursos, el rector renunciante planteó que se iniciaba un nuevo período en la vida de la Universidad y que había que tener "fe en el engrandecimiento de esta casa." Para eso pedía el trabajo de todos "en un ambiente de lealtad y de concordia, deponiendo desconfianzas y recelos que nunca serán fundamento de obra duradera." 42

En la calle los grupos de estudiantes se ubicaron en las puertas de sus locales. Al pasar el ex rector Martínez, fue el grupo de la Unión Universitaria el que lo "vivó estruendosamente." Mientras tanto, los de la Federación Universitaria saludaban con alborozo a los profesores que se retiraban de la casa de estudios y a las personas que circulaban por la calle. Los catedráticos de las dos facultades pusieron sus renuncias a disposición de la intervención y ésta, a través de un decreto, designó a los nuevos secretarios. Se trataba de los Profesores Amadeo Gervasoni, que se venía desempeñando como Director de Instrucción Pública de la provincia y Antenor Ferreira, Inspector General de Escuelas de la provincia. El diario llamaba la atención sobre el dato ya que ninguno de los dos era abogado y expresaba que ello se podía deber a que "el señor interventor ha procedido así por la dificultad de encontrar un abogado que no esté embanderado en este conflicto." 43

La renuncia del rector y de los profesores de la universidad de Santa Fe fueron tomadas por los dirigentes estudiantiles como un triunfo indiscutido de sus

\footnotetext{
${ }^{38}$ Santa Fe, Santa Fe, 14 de mayo de 1919, p. 2

39 Alejandro Grüning Rosas, Creación de la Universidad Nacional del Litoral. Apéndice documental complementario...0p. Cit. p. 64.

${ }^{40}$ Santa Fe, Santa Fe, 15 de mayo de 1919, p. 2.

41 Ibídem.

42 Ibídem.

43Ibídem.
} 


\section{Eliana Bertero y José Larker}

acciones. A modo de celebración, la Federación Universitaria organizó un acto en el Teatro Municipal el domingo 18 de mayo. El momento artístico estuvo a cargo de una academia de declamación y luego hicieron uso de la palabra Grüning Rosas, quien presentó al delegado de los universitarios de Córdoba, Cortés Plá, y Humberto Gambino hizo lo propio con el de la Federación Universitaria Argentina, Ubaldo Isnardi.

A pesar de estos logros en los días siguientes, los estudiantes universitarios continuaron con sus demandas, interviniendo en el espacio público a través de conferencias en asociaciones civiles y gremiales ${ }^{44}$ y de la movilización callejera.

El 28 de mayo se realizó un mitin que recibió la adhesión de numerosas instituciones como la Asociación Gremial de Maestros, la Federación Obrera, la Unión Ferroviaria, el Centro Sindical de Cocineros y Pasteleros, la Sociedad Unión Hebrea, el Centro Socialista, Biblioteca Monteagudo, el Centro del Libre Pensamiento y los Centros de Estudiantes del Colegio Nacional y de la Escuela Industrial. Según lo expresa el diario de campaña de los estudiantes de Santa Fe, en esa oportunidad se aprobó un documento en el que se manifestaba, entre otras cosas, "su inquebrantable fe en luchar contra el despotismo metido en la escuela y la incapacidad enseñoreada de la cátedra, llena de la más resuelta y firme decisión de despertar en una intensa sacudida las actividades de un pueblo." Planteaban que ellos enarbolaban el espíritu de "Mariano Moreno contra los pelucones de la primera junta." ${ }^{45}$ Las críticas expuestas en este documento cuestionaban una vez más a las autoridades y al profesorado, vinculados a las tendencias conservadoras y opuestas a la transformación de la institución.

El mitin había comenzado con una marcha por calle San Martín. En el trayecto se produjeron dos actos de hostilidad, uno de ellos frente al Club del Orden, pero sin pasar a mayores. Los manifestantes cubrieron un amplio espacio a lo largo de las calles San Jerónimo y 3 de febrero. Desde la tribuna hablaron los delegados de la Universidad de Córdoba Enrique Barros, Ismael Bordabehere y Emilio Biagosch, el delegado de la Federación Universitaria Argentina Ubaldo Isnardi, Armando Molinas, Augusto Morisot y Pablo Vrillaud como Presidente de la Federación Universitaria de Santa Fe. Al concluir se invitó a los presentes a asistir al local de la Federación donde volvieron a producirse discursos y adhesiones que reivindicaban la lucha del movimiento reformista santafesino, como la que hiciera llegar, Arturo Capdevilla:

Diga en mi nombre, hermano Enrique [Barros] (...) a esos valientes amigos de la ciudad de Santa Fe que han hecho bien en alzarse contra Loyola y Fernando VII. Dígales que si les llaman abanderados de la

\footnotetext{
${ }^{44}$ Como las realizadas en la Federación Obrera, en la Biblioteca Emilio Zola y en la Sociedad Cosmopolita, entre otras. Ángel Caballero Martín. La Universidad..., Op. cit, p. 125.

${ }^{45}$ Diario de campaña de los estudiantes de Santa Fe por la reforma Universitaria. En Gabriel Del Mazo, La Reforma Universitaria: Documentos complementarios que se refieren a la acción directamente social del Movimiento estudiantil argentino (1918-1921), Federación Universitaria de Buenos Aires, Buenos Aires, 1927, Tomo V, p. 48-49
} 


\section{El movimiento estudiantil santafesino y sus estrategias de intervención colectiva en tiempos de lucha por la reforma universitaria y la creación de la Universidad Nacional del Litoral (1918 y 1919)}

bandera roja, respondan así: "Nuestro rojo es el rojo del gorro frigio". Aquí no hay otra bandera roja que la que resulta de la negra jesuítica, ardiendo en llamas al viento de la libertad. ${ }^{46}$

Al momento de la desconcentración se produjeron algunos incidentes provocados por un grupo de personas que llevaban una bandera Argentina. Según relata la prensa local, de los episodios participaron integrantes del Regimiento12 de infantería "con el propósito de desvirtuar el motivo de la asamblea pública". ${ }^{7}$ Por estos incidentes la Federación Universitaria hizo pública una declaración y envió al Presidente de la República y a los Ministros del Interior, Instrucción Pública y de Guerra un telegrama de protesta denunciando a los oficiales del 12 de infantería. ${ }^{48}$ En los días siguientes, los universitarios santafesinos, recibieron una comunicación de la Federación Universitaria Argentina en la que expresaban que ante el conflicto por el que estaba atravesando la Universidad de Santa Fe resolvía solicitar al Congreso de la Nación atender al asunto y apoyar la actitud de la Federación Universitaria de Santa Fe. ${ }^{49}$ También se recibieron notas de adhesión de la Federaciones Universitarias de Buenos Aires, de Córdoba, de La Plata y de Tucumán.

Las repercusiones del conflicto y las adhesiones que recibían los estudiantes, dejaban en evidencia que la cuestión universitaria santafesina había sido adoptada como propia tanto por grupos intelectuales como por sectores sindicales, y había desbordado el plano estrictamente local.

En este contexto, el 3 de junio en el Congreso de la Nación, la comisión de Instrucción Pública dio ingreso al proyecto de Ley, aprobando una moción de preferencia para su tratamiento. Durante esos días se sumaron los pedidos para la inmediata sanción de la ley. En ese sentido, enviaron sus solicitudes a la Cámara de Diputados de la Nación el Gobernador de la Provincia de Santa Fe, la Federación Universitaria Argentina, la Federaciones Universitarias de Santa Fe y Buenos Aires, así como diversas instituciones de Santa Fe y Rosario. En Buenos Aires lo delegados de la Federación Universitaria de Santa Fe (Alejandro Grüning Rosas, José Rubio Olssen, Francisco J. Garo y Domingo Albornoz) realizaron gestiones en diversos ámbitos y con el mismo propósito. En la sesión del 11 de junio comenzó el tratamiento de los proyectos, las discusiones fueron arduas y se extendieron en el tiempo.

En Santa Fe el interventor de la Universidad convocó, para el 1 de agosto, a los estudiantes de las Facultades de Derecho y de Farmacia y Obstetricia con motivo de la elección de los delegados que en compañía de los profesores constituirían la Asamblea Universitaria. Sin lugar a dudas se trataba de un acontecimiento de

\footnotetext{
${ }^{46}$ Celestino Lanteri, Pablo Vrillaud. Líder de la juventud. Santa Fe, Centro de Publicaciones de la UNL - Centro de Estudiantes de Derecho, 1995. p. 113.

${ }^{47}$ Santa Fe, Santa Fe, 30/05/1919, p. 2.

48 Diario de campaña de los estudiantes de Santa Fe por la reforma Universitaria. Mayo 29. En Gabriel Del Mazo, La Reforma Universitaria..., Op. Cit., Tomo V, p. 48-49.

49 Ibídem.
} 


\section{Eliana Bertero y José Larker}

importancia. Por primera vez los estudiantes iban a tener injerencia en el gobierno de la Universidad. Participaron del acto todos los estudiantes universitarios y las listas de delegados propuestas por la Federación obtuvieron el triunfo por amplio margen de votos..$^{50}$ El día 18 de agosto se realizaron las asambleas en las que se eligieron los Decanos de las dos facultades, así como también los Consejeros Directivos. En todos los casos las listas de la Federación Universitaria lograron el triunfo. ${ }^{51}$ Ante los resultados, un numeroso grupo de profesores presentó su renuncia, inclusive algunos que habían logrado cargos directivos por designación de los mismos estudiantes. Según Caballero Martín se trataba de dejar acéfalo al instituto e impedir el proceso de cambio. La Federación Universitaria dio a conocer una resolución por la que se declaraba partidaria de aceptar las renuncias de los profesores y expresó que contaba "con el concurso espontaneo de numerosos y conspicuos profesionales" para "mantener el Instituto Superior de cultura de Santa $\mathrm{Fe}^{\prime}$ y que en caso de que se intentara clausurar la Universidad estaba dispuesta a defenderla "propiciando ante el pueblo de Santa Fe un amplio movimiento de protesta." 52

Mientras eso sucedía en Santa $\mathrm{Fe}$, las organizaciones estudiantiles universitarias siguieron presionando a los diputados a través de las notas que enviaban solicitando la aprobación del proyecto de Ley por el que se creaba la Universidad Nacional del Litoral. Ésta se produjo en Diputados el 27 de agosto y pocos días después ingresó al Senado. Una vez más la Federación Universitaria Argentina, la Sociedad Amigos de la Infancia y sociedades culturales, industriales, comerciales y estudiantiles de Santa Fe, Rosario y Paraná, se dirigieron a la cámara urgiendo su pronto tratamiento. Un mes después se presentó el despacho de la comisión respectiva y se dio por aprobada la ley. ${ }^{53}$

Mientras tanto en Santa Fe el interventor aceptaba las renuncias presentadas por los docentes y cubría las vacantes según lo establecido por los estatutos. Por otro lado, el 20 de septiembre se constituyó el Consejo Directivo de la Facultad de Farmacia y Obstetricia y el 25 del mismo mes, el de la Facultad de Derecho y Ciencias Sociales. Una vez más, los profesores propuestos por la Federación fueron electos. De esa manera, la Federación Universitaria siguió imponiéndose durante el periodo que duró la intervención.

El diario de campaña de los estudiantes dice que el día 27 de septiembre se festejó ruidosamente la noticia de la sanción definitiva de la Ley de creación de la Universidad Nacional del Litoral. Cuatro días después la Federación Universitaria resolvió ponerle punto final a la huelga que mantenían. Se consideró que la lucha no había terminado pero creían prudente reemprender la tarea suspendida "no por

\footnotetext{
50 Ángel Caballero Martín, La Universidad..., Op. Cit, p. 138.

51 Diario de campaña de los estudiantes de Santa Fe por la reforma Universitaria. En Gabriel Del Mazo, La Reforma Universitaria..., Op. Cit.,p. 51.

${ }^{52}$ Ángel Caballero Martín, La Universidad..., Op. Cit, p. 142 y 143.

53 El presidente Hipólito Yrigoyen promulgó la ley el 17 de octubre de 1919 bajo el Número 10861.
} 


\section{El movimiento estudiantil santafesino y sus estrategias de intervención colectiva en tiempos de lucha por la reforma universitaria y la creación de la Universidad Nacional del Litoral (1918 y 1919)}

puro motivo de egoísmo, sino porque así lo reclama la moralidad y el decoro de los estudiantes y la seriedad docente y administrativa de la Universidad."54

En marzo de 1920 el presidente Hipólito Yrigoyen encargó al Ministro de Justicia e Instrucción Pública de la Nación José S. Salinas la organización de la Universidad en función de lo estipulado por la ley. Concluido los trabajos de organización, el 12 de abril de 1922 Yrigoyen nombró rector, hasta el momento que se eligieran las autoridades de acuerdo a lo establecido por los estatutos, a José Avalos y una semana después el Ministro Salinas se trasladó a Santa Fe para proceder a la inauguración de la Universidad Nacional del Litoral. El movimiento estudiantil había logrado su objetivo y este hecho ponía de manifiesto el rol decisivo de sus acciones en la concreción de la nueva universidad.

\section{Consideraciones finales}

Durante la década de 1910, los jóvenes universitarios santafesinos fueron delineando y consolidando un movimiento de carácter reformista. Desde sus inicios, asumió posiciones sobre cuestiones sociales y políticas, que excedían la esfera universitaria. Como hemos visto, los jóvenes respaldaron sus argumentos y acciones en diversos escritos, tales como los proyectos de universidad, notas y artículos difundidos en la prensa local y en órganos propios como la Gaceta Universitaria de Santa Fe.

En el contexto santafesino de principios de siglo XX, el conflicto universitario fue parte de la batalla por la modernización de la sociedad, la cultura y la política provinciales. En ella, los estudiantes universitarios se constituyeron en un actor político que intervino activamente en la esfera pública local y nacional, articulando redes de solidaridad con asociaciones liberales que intervinieron fuertemente en el proceso de modernización. En el mismo sentido, los locales de las federaciones obreras y de trabajadores, se convirtieron en tribuna de los discursos y acciones de los estudiantes reformistas. Sin duda, jugó un papel importante en la configuración del movimiento reformista, la llegada a la universidad de los jóvenes de clase media. Para la mayoría de éstos, como se advierte en las trayectorias de Pablo Vrillaud, Mariano Tissembaun y Horacio Varela, la militancia en el movimiento reformista fue el espacio en el que hicieron sus primeras experiencias políticas. ${ }^{55}$

Los acontecimientos de Córdoba y la creación de la Federación Universitaria Argentina, dieron un nuevo impulso a las demandas y acciones del movimiento estudiantil santafesino. La posibilidad de tramar vínculos permanentes a través de la organización gremial, con los estudiantes universitarios en el ámbito nacional,

\footnotetext{
54 Diario de campaña de los estudiantes de Santa Fe por la reforma Universitaria. En Gabriel Del Mazo, La Reforma Universitaria..., Op. Cit., p. 54.

${ }^{55}$ Sobre la cultura reformista y los inicios de las experiencias políticas de los jóvenes universitarios recuperamos los aportes de Natalia Bustelo, La reforma universitaria desde sus grupos y revistas...0p. Cit.
} 


\section{Eliana Bertero y José Larker}

tuvo un peso indudable en la sanción del proyecto de Universidad Nacional del Litoral.

En ese clima, el conflicto universitario profundizó las disputas entre la juventud reformista y los grupos más conservadores, identificados con las autoridades y algunos profesores de la universidad provincial. En el transcurso de la contienda, la joven generación se encontró en pugna con una elite discorde con la ampliación de la ciudadanía y la movilidad social que posibilitaba el acceso de los sectores medios, a los bienes educativos. En este sentido, como subraya Oscar Terán, en sus discursos y acciones, la juventud reformista se autoconstruye como una nueva generación que ha venido a romper con la anterior, en otras palabras, como agente privilegiado del cambio social. ${ }^{56}$

\section{Bibliografía}

Alejandro Grüning Rosas Creación de la Universidad Nacional del Litoral (Crónica Retrospectiva). Apéndice documental complementario, Buenos Aires, 1940 Creación de la Universidad Nacional del Litoral.

Crónica retrospectiva. Santa Fe, Imprenta de la Universidad. 1940

Ángel Caballero Martín, La Universidad de Santa Fe, Imprenta de la Universidad, Santa Fe, 1931

Bernardo Carrizo, "Temas y debates en tiempos de cambio. La legislatura de Santa Fe, 1912-1916". XII Jornadas Interescuelas/Departamentos de Historia. Facultad de Humanidades y Centro Regional Universitario Bariloche. Universidad Nacional del Comahue, San Carlos de Bariloche, 2009.

Celestino Lanteri, Pablo Vrillaud. Líder de la juventud. Santa Fe, Centro de Publicaciones de la UNL - Centro de Estudiantes de Derecho, 1995.

Cesar Tcach, De la monotonía de los claustros a la polifonía de las ideas: introducción a la Gaceta Universitaria. En La Gaceta Universitaria 1918-1919. EUDEBA, Buenos Aires, 2008

Darío, Macor, "Reforma política, reforma del Estado. La ciudad de Santa Fe en los años veinte y treinta. Espacios de constitución de lo político", en Devoto, Fernando y Ferrari, Marcela (comps.), La construcción de las democracias rioplatenses: proyectos institucionales y prácticas políticas, 1900-1930, Buenos Aires, UNMDP/Biblos. 1994.

Eduardo Zimmermann, "Los intelectuales y el reformismo liberal", en Desarrollo Económico, № 124, 1992.

Eliana Bertero, "Estado, política y educación. Ideas e instituciones en torno a la laicización", en Bernardo Carrizo y Juan Cruz Giménez (coordinadores), La política en las tramas educativas, Paraná, La Hendija, 2017.

${ }^{56}$ Oscar Terán. Historia de la ideas en la Argentina. Diez lecciones iniciales, 1810-1980, Buenos Aires, Siglo XXI, 2008. 


\section{El movimiento estudiantil santafesino y sus estrategias de intervención colectiva en tiempos de lucha por la reforma universitaria y la creación de la Universidad Nacional del Litoral (1918 y 1919)}

Eliana Bertero, Valeria Pini, Matías Vicentín, Logia Armonía. Masones y librepensadores en la esfera pública, Santa Fe, Ediciones UNL, 2015.

Gabriel Del Mazo, La Reforma Universitaria, documentos relativos al movimiento estudiantil en las universidades de Córdoba y Buenos Aires, Federación universitaria de Buenos Aires, Buenos Aires, 1927, Tomo llI

-La Reforma Universitaria: Documentos complementarios que se refieren a la acción directamente social del Movimiento estudiantil argentino (1918-1921), Federación Universitaria de Buenos Aires, Buenos Aires, 1927, Tomo $\mathrm{V}$

José Larker, "La Universidad de Santa Fe, la formación de abogados y sus concepciones acerca del Derecho." Ponencia presentada en las I Jornadas de Historia de la Universidad en la Argentina, Santa Fe, Universidad Nacional del Litoral, 2008

"El movimiento estudiantil universitario santafesino: acción y participación en el proceso de creación de la Universidad Nacional del Litoral", en Natalia Vega y Luciano Alonso (Compiladores), Lugares de lo colectivo en la historia local. Asociaciones, trabajadores y estudiantes de la zona santafesina. Santa Fe, María Muratore Ediciones, 2017.

Luk Boltanski, L'espacepositionnel. Multiplicité des positions institutionnelles et habitus de clase. En Revuefran ̧aise de sociologie, XIV, 1973. Traducción Nora Vega. Manuel Menchaca, "La Universidad Nacional del Litoral. Antecedentes de su creación", en Revista Universidad, N 48, Santa Fe, UNL, 1961

Natalia Bustelo, La reforma universitaria desde sus grupos y revistas: Una reconstrucción de los proyectos y las disputas del movimiento estudiantil porteño de las primeras décadas del siglo XX (1914-1928). Tesis de posgrado. Universidad Nacional de La Plata. Facultad de Humanidades y Ciencias de la Educación. En Memoria Académica. $2015 . \quad$ Disponible en: http://www.memoria.fahce.unlp.edu.ar/tesis/te.1307/te.1307.pdf

Oscar Terán, Historia de la ideas en la Argentina. Diez lecciones iniciales, 1810-1980, Buenos Aires, Siglo XXI, 2008.

Osvaldo Graciano, Entre la torre de marfil y el compromiso político. Intelectuales de izquierda en la Argentina 1918-1955, Bernal, editorial UNQ, 2008.

Pablo Buchbinder ¿Revolución en los claustros?: la reforma universitaria de 1918, Buenos Aires, Sudamericana, 2008.

Portantiero, Juan Carlos. Estudiantes y política en América Latina (1918-1938). El proceso de la Reforma Universitaria. Buenos Aires: Siglo XXI, 1978.

Raúl Villarroel, "Santa Fe", RACP, Tomo X, Buenos Aires, mayo de 1915.

Recibido: 20/05/2018

Evaluado: 15/07/2018

Versión Final: 30/08/2018 